SHORT REPORT

\title{
Colonic transit time and rectoanal videomanometry in Parkinson's disease
}

\author{
R Sakakibara, T Odaka, T Uchiyama, M Asahina, K Yamaguchi, T Yamaguchi, \\ T Yamanishi, T Hattori
}

Background: Constipation is a prominent lower gastrointestinal tract dysfunction that occurs frequently in Parkinson's disease (PD).

Objective: To investigate colonic transport and dynamic rectoanal behaviour during filling and defecation in patients with PD.

Methods: Colonic transit time (CTT) and rectoanal videomanometry analyses were performed in 12 patients with PD 110 men and 2 women; mean age, 68 years, mean duration of disease, five years; mean Hoehn and Yahr grade, 3; decreased stool frequency $\mid<3$ times a week) in six, difficulty in stool expulsion in eight) and 10 age matched normal control subjects 17 men and 3 women; mean age, 62 years; decreased stool frequency in two, difficulty in stool expulsion in two).

Results: In the PD patients, CTT was significantly prolonged in the rectosigmoid segment $(p<0.05)$ and total colon $(p<0.01)$ compared with the control subjects. At the resting state, anal closure and squeeze pressures of PD patients were lower than those in control subjects, though not statistically significant. However, the PD patients showed a smaller increase in abdominal pressure on coughing $(p<0.01)$ and straining $(p<0.01)$. The sphincter motor unit potentials of the patients were normal. During filling, PD patients showed normal rectal volumes at first sensation and maximum desire to defecate, and normal rectal compliance. However, they showed smaller amplitude in phasic rectal contraction $(p<0.05)$, which was accompanied by an increase in anal pressure that normally decreased, together with leaking in two patients. During defecation, most PD patients could not defecate completely with larger post-defecation residuals $(p<0.01)$. PD patients had weak abdominal strain and smaller rectal contraction on defecation than those in control subjects, though these differences were not statistically significant. However, the PD patients had larger anal contraction on defecation $(p<0.05)$, evidence of paradoxical sphincter contraction on defecation (PSD).

Conclusions: Slow colonic transit, decreased phasic rectal contraction, weak abdominal strain, and PSD were all features in our PD patients with frequent constipation.

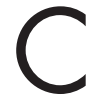
nstipation is a prominent lower gastrointestinal (GI) tract feature in patients with Parkinson's disease (PD) and can appear earlier than motor disorder. ${ }^{1}$ Constipation not only impairs the quality of life of the patients, but also affects dopaminergic drug absorption, or may lead to an emergency colonic pseudo-obstruction. ${ }^{2}$ The incidence rate of decreased stool frequency ( $<3$ times a week) in PD patients is over $50 \%$ (range $29 \%-67 \%$ ), and that of difficulty in stool expulsion in $57 \%-67 \%$, both of which are significantly higher than in the normal population. ${ }^{3-5}$ The lower GI tract function primarily consists of (1) colonic transport of the bowel content to the anorectum, (2) transient anorectum reservoir, and (3) defecation from the anorectum. However, the dynamic behaviour of the lower GI tract in PD patients is not fully understood. The purpose of this study is to present the results of our colonic transit time and rectoanal videomanometry analyses in patients with PD.

\section{METHODS}

We recruited 12 patients with $\mathrm{PD}$, who met the clinical diagnostic criteria ${ }^{6}$ including MRI scan and responded well to levodopa. Because it is difficult for PD patients to stop taking levodopa, we assessed the patients under medication in this study, although dopaminergic drugs are reported to have facilitatory effects on the lower GI tract function. ${ }^{78}$ At the time of the study their average daily levodopa (with a combination of carbidopa) requirement was $200 \mathrm{mg}$ (range 100-300 mg). None of the patients was taking anti-cholinergic agents. The group comprised 10 men and 2 women, mean age 68 years (50-75 years), mean duration of illness five years ( $1-8$ years). The severity of illness ranged from 1 to 3 on the Hoehn and Yahr scale, with a median value of 3. A detailed questionnaire on the pelvic organ function ${ }^{3}$ showed that 10 patients had bowel symptoms, including decreased stool frequency $(<$ once per three days) in six and difficulty in expulsion in eight, but neither fecal incontinence nor diarrhoea in any of the patients. The control group consisted of 10 normal subjects: seven men and three women, mean age 62 years ( $42-75$ years). Two of the controls had decreased stool frequency, two had difficulty in expulsion, and none had fecal incontinence or diarrhoea. None of the PD patients or controls had abnormalities in the blood chemistry (including blood sugar) or urine analysis.

The colonic transit study was performed using the repetitive ingestion method. ${ }^{9}$ We asked the subjects to ingest a small test capsule once a day for six days shortly after breakfast. The capsule contained 20 circular radio-opaque markers. A week later, we took a plain abdominal rdiograph. The markers were counted in three segments of the large bowel: the right colon, the left colon, and the sigmoid colon and rectum. One marker corresponds to 1.2 hours (20 markers for 24 hours) of transit time. We counted the number of markers in each segment of the large bowel. We multiplied these numbers by 1.2 to derive the colonic transit time (CTT) in that part of the colon-that is, the right CTT, left CTT, and rectosigmoid CTT, respectively, and took the sum of these transit times as the total CTT.

The videomanometry was performed using a Urovision computer (Lifetech, USA) and an electromyographic computer (Neuropack $\Sigma$; Nihon Kohden, Tokyo, Japan). Firstly, a triple lumen 8 French gauge catheter (for use with contrast

Abbreviations: PD, Parkinson's disease; CTT, colonic transit time; PSD, paradoxical sphincter contraction on defecation; GI, gastroinestinal 


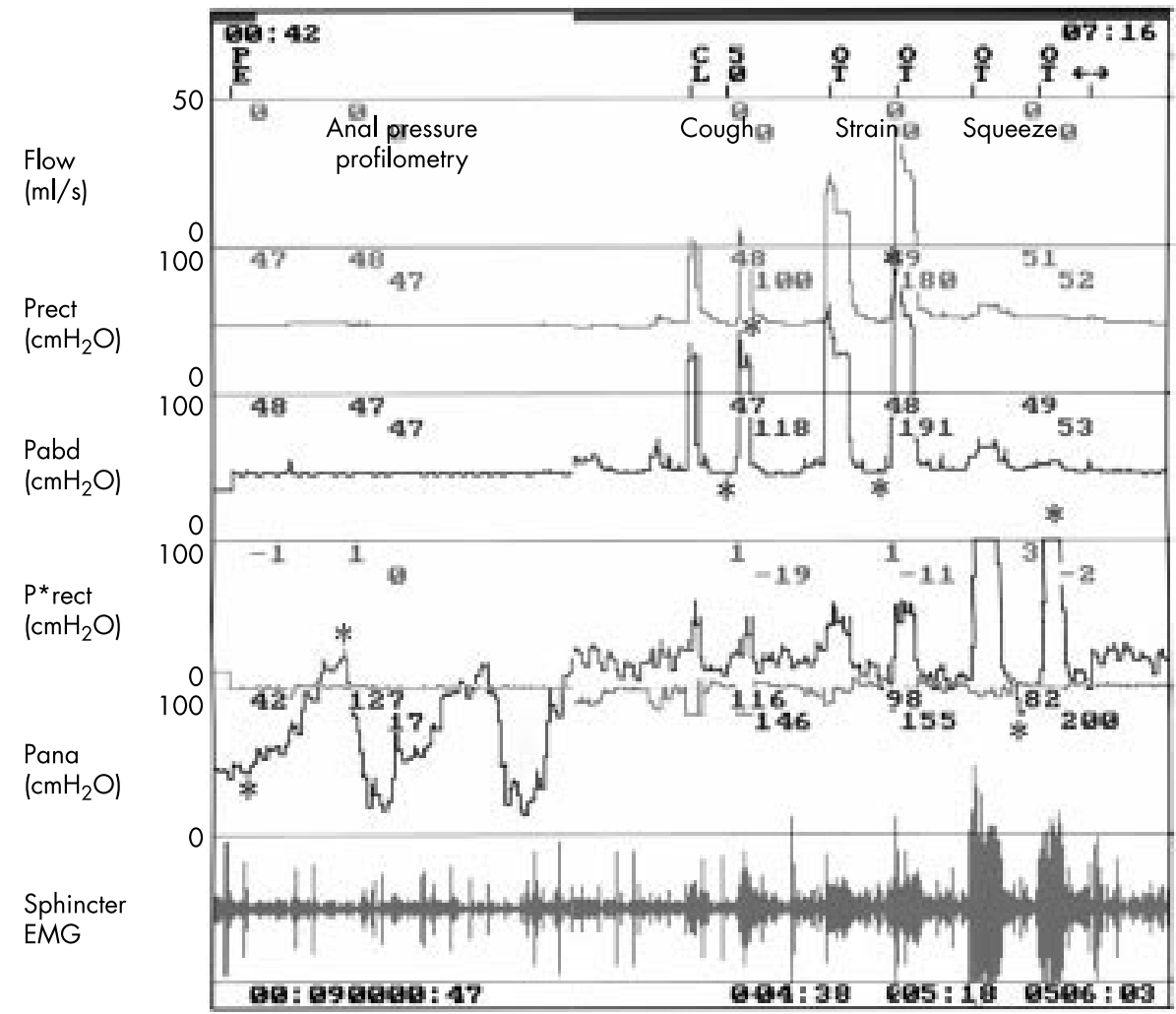

Figure 1 Resting state manometry (control subject). Prect, rectal pressure; Pabd, abdominal (bladder) pressure; $\mathrm{P}$ *ect, differential rectal pressure = Prect-Pabd; Pana, anal pressure, EMG, electromyography. This subject had normal anal closure pressure $>40 \mathrm{~cm} \mathrm{H}_{2} \mathrm{O}$ and anal pressure increase on squeezing $>50 \mathrm{~cm} \mathrm{H}_{2} \mathrm{O}$ (Pana). On coughing and straining, his abdominal pressure increased significantly (Pabd).

medium infusion, rectal, and anal pressures) was inserted into the anus. At the tip of the catheter a small ( $1 \mathrm{~cm}$ diameter) ultra-elastic balloon was attached, which was filled with a minimum amount of water. Another 8 French gauge catheter (for use with abdominal pressure) was inserted into the bladder. We performed static anal manometry by pulling the catheter from inside the rectum $(2 \mathrm{~cm} / \mathrm{min})$ throughout the anal canal while infusing sterile water at a rate of $1 \mathrm{ml} / \mathrm{min}$. The anal pressure showed a maximum value at the external sphincter, where the radio-opaque marker attached to the anal luminal orifice was visualised-that is, 2.0 to $3.0 \mathrm{~cm}$ below the rectal base under an $x$ ray fluoroscope. The normal value of the anal closure pressure, which is the difference between the maximum anal pressure and the rectal pressure, is $>40 \mathrm{~cm}$ $\mathrm{H}_{2} \mathrm{O} .{ }^{10}$ We then measured an increase in abdominal and anal pressures on coughing, straining (Valsalva manoeuver), and squeezing as much as possible. The normal value of anal pressure increase on squeezing is $>50 \mathrm{~cm} \mathrm{H}_{2} \mathrm{O}^{10}$ A concentric needle electrode was inserted into the external anal sphincter. Neurogenic sphincter EMG was diagnosed when at least one of the following abnormalities was seen: a mean duration of motor unit potentials $>10 \mathrm{~ms}$; and a percentage of motor unit potentials with a duration $>10 \mathrm{~ms}$ greater than $20 \% .{ }^{11}$ In addition, we analysed the amplitude and the number of phases of each motor unit potential. Secondly, we performed medium fill $(50 \mathrm{ml} / \mathrm{min})$ videomanometry in a sitting position with infusing contrast medium (20\% amidotrizoic acid) into the rectum, simultaneously recording the rectal pressure, which is the difference between the naive rectal pressure and abdominal (bladder) pressure, in addition to anal pressure, sphincter EMG activity, fecal flow, and fluoroscopic images of the sigmoid colon, rectum, and anus. During the procedure, none of the subjects had abdominal discomfort or pain, as sometimes occur in cases of diarrhoea with abnormal bowel contraction. Rectal compliance, reflecting a tonic increase of the rectal pressure, is defined as $\Delta$ infused volume/ $\Delta$ rectal pressure $\left(\mathrm{ml} / \mathrm{cm} \mathrm{H}_{2} \mathrm{O}\right) .{ }^{12}$ If the subject had concurrent phasic contractions, we used the bottom line of the final phasic contraction for measuring the rectal compliance. All patients gave informed consent before the studies. Statistical analysis was made by Student's $t$ test.

\section{RESULTS}

\section{Colonic transit study}

In the PD patients, the mean right CTT of 28.8 minutes and left CTT of 20.5 minutes were not significantly prolonged compared with those in the control subjects (6.9 minutes and 14.1 minutes, respectively). However, the rectosigmoid CTT of 34.0 minutes $(p<0.05)$ and total CTT of 82.4 minutes $(\mathrm{p}<0.01)$ in the PD patients were significantly prolonged compared with those in the control subjects ( 18.0 minutes and 39.0 minutes, respectively).

\section{Rectoanal videomanometry}

Resting

All control subjects showed anal closure pressure $>40 \mathrm{~cm} \mathrm{H}_{2} \mathrm{O}$ and anal pressure increase on squeezing $>50 \mathrm{cmH}_{2} \mathrm{O}$ (fig l). The anal closure pressure of the PD patients $\left(48.4 \mathrm{~cm} \mathrm{H}_{2} \mathrm{O}\right)$ was lower than that of control subjects $\left(63.8 \mathrm{~cm} \mathrm{H} \mathrm{H}_{2} \mathrm{O}\right)$, although the difference was not statistically significant. Similarly, the anal pressure increase on squeezing of the PD patients $\left(88.3 \mathrm{~cm} \mathrm{H}_{2} \mathrm{O}\right)$ was slightly, but not significantly, lower than that of control subjects $\left(96.1 \mathrm{~cm} \mathrm{H}_{2} \mathrm{O}\right)$. The PD patients showed a less pronounced abdominal pressure increase on coughing $\left(62.9 \mathrm{~cm} \mathrm{H}_{2} \mathrm{O}\right)(\mathrm{p}<0.01)$ and straining $\left(29.2 \mathrm{~cm} \mathrm{H} \mathrm{H}_{2} \mathrm{O}\right)(\mathrm{p}<0.01)$ than the control subjects $(98.4 \mathrm{~cm}$ $\mathrm{H}_{2} \mathrm{O}$ and $71.0 \mathrm{~cm} \mathrm{H}_{2} \mathrm{O}$, respectively). The PD patients had sphincter motor unit potentials with a mean duration of 6.3 $\mathrm{ms}$, a percentage of units with a duration over $10 \mathrm{~ms}$ of $7 \%$, an amplitude of $524 \mu \mathrm{V}$, and a phase number of 3.5. These figures 
were not different from those in the control subjects $(7.0 \mathrm{~ms}$, $13 \%, 255 \mu \mathrm{V}$, and 3.0, respectively).

\section{Rectal filling}

The PD patients had slightly larger rectal volume at first sensation $(131.1 \mathrm{ml})$ and maximum capacity $(345.6 \mathrm{ml})$ than those in the control subjects $(127.8 \mathrm{ml}$ and $302.2 \mathrm{ml}$, respectively), although the differences were not statistically significant. The rectal pressure of the subjects gradually increased along with rectal filling both tonically and phasically (fig 2A). The spontaneous phasic contraction became apparent just before the first sensation. After that, radio-opaque infusant tended to exceed the rectosigmoid junction. The PD patients had the same, high rectal compliance $\left(73.1 \mathrm{ml} / \mathrm{cm} \mathrm{H}_{2} \mathrm{O}\right)$ as the control subjects $(69.3$ $\mathrm{ml} / \mathrm{cm} \mathrm{H}_{2} \mathrm{O}$ ). However, the amplitude of the phasic rectal contraction $\left(12.2 \mathrm{~cm} \mathrm{H}_{2} \mathrm{O}\right)$ in the PD patients was less than that in the control subjects $\left(22.2 \mathrm{~cm} \mathrm{H}_{2} \mathrm{O}\right)(\mathrm{p}<0.05)$. Spontaneous phasic anal contraction was also observed. When the rectal pressure increased, the anal pressure in normal subjects decreased $\left(-11.2 \mathrm{~cm} \mathrm{H}_{2} \mathrm{O}\right)$ together with the radiographical open anal neck and urgency sensation. However, sphincter EMG activity did not cease, and none of the control subjects showed leaking. On the other hand, when the rectal pressure increased, the anal pressure in the PD patients increased (14.4 $\mathrm{cm} \mathrm{H}_{2} \mathrm{O}$ ) (fig 2C), and two patients leaked rectal content.

\section{Defecation}

Most PD patients could not defecate completely and showed post-defecation residuals of $133 \mathrm{ml}$, a volume significantly larger than that in the control group $(p<0.01)$. Four of the control subjects showed a rectal pressure increase on defecation, after the phasic rectal contraction during filling (rectal contraction type defecation) (fig 2A). The other subjects defecated only with abdominal strain, particularly those who had smaller phasic rectal contraction during filling (strain type defecation) (fig 2B). Overall, both abdominal and

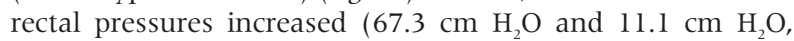
respectively) in the control subjects. Similarly, two of the PD patients had the rectal pressure increase on defecation. The other patients defecated only with abdominal strain. However, the increases in abdominal pressure $\left(53.9 \mathrm{~cm} \mathrm{H}_{2} \mathrm{O}\right)$ and rectal pressure $\left(4.4 \mathrm{~cm} \mathrm{H}_{2} \mathrm{O}\right)$ in the PD patients were less than those in the control subjects, although these differences did not reach the level of statistical significance. In the control subjects, radiographical opening of the anal canal was seen in both types of defecation. Strain type defecation was also accompanied by a descent of the rectal base. Sphincter EMG relaxation and anal pressure decrease were seen in rectal contraction type defecation, but not in strain type defecation, and overall the anal pressure increased moderately ( $19.6 \mathrm{~cm} \mathrm{H}_{2} \mathrm{O}$ ) during defecation. However, the PD patients showed an incomplete opening of the anal canal with little descent of the rectal base. The anal pressure increase on defecation in the patients $\left(49.6 \mathrm{~cm} \mathrm{H}_{2} \mathrm{O}\right.$ ) was significantly larger than that in the control subjects $(\mathrm{p}<0.05)$ (fig $2 \mathrm{C})$, together with an increase in the sphincter EMG activity.

\section{DISCUSSION}

This study showed prolonged CTT in the PD patients. In particular, the rectosigmoid CTT $(\mathrm{p}<0.01)$ and total CTT $(\mathrm{p}<0.05)$ were significantly prolonged in $\mathrm{PD}$ patients, in agreement with previous reports. ${ }^{13}{ }^{14}$ We therefore concluded that rectosigmoid CTT made the greatest contribution to the prolonged total CTT of the PD patients. The slow colonic transit reflects decreased contraction of the colonic smooth muscles, which is the major cause of decrease in stool frequency in PD patients. The slow colonic transit is likely to reflect both central and peripheral abnormality in PD. Experimental studies have shown that the basal ganglia primarily
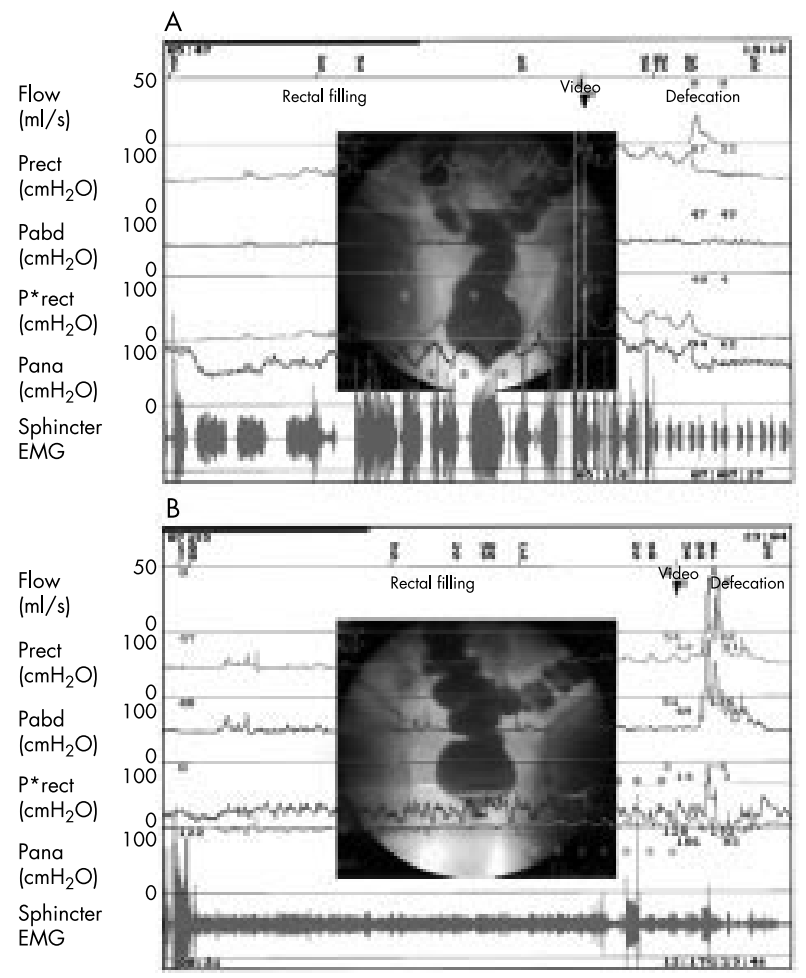

$\mathrm{C}$

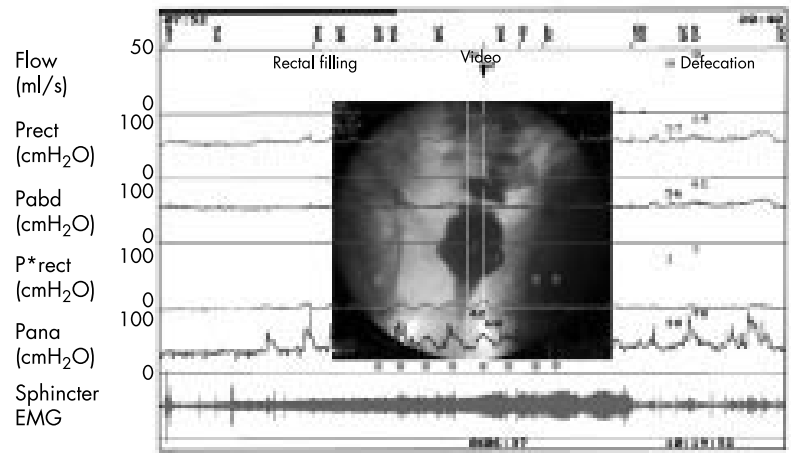

Figure 2 Filling and defecation videomanometry (two control subjects; A, B, and a Parkinson's disease patient; C). Flow: fecal flow; Prect, rectal pressure, Pabd, abdominal (bladder) pressure; $\mathrm{P}^{*}$ rect, differential rectal pressure $=$ Prect-Pabd; Pana, anal pressure; EMG, electromyography; SI, start of infusion; FS, first sensation; El, end of infusion (maximum capacity); VD, defecation. During filling, the first subject $(A)$ had spontaneous phasic rectal contractions ( $\mathrm{P}^{*}$ rect). Spontaneous phasic anal contractions were also observed (Pana). When the rectal pressure increased, the anal pressure tended to decrease together with radiographical open anal neck (video). The second subject (B) had smaller phasic rectal contractions. Anal pressure in this subject at start of infusion was $122 \mathrm{~cm} \mathrm{H}_{2} \mathrm{O}$. The Parkinson's disease patient $(\mathrm{C})$ also had smaller phasic rectal contractions. However, when the rectal pressure increased, the anal pressure tended to increase with marked open anal neck (video), and this patient leaked rectal content intermittently (flow). During defecation, the first subject showed as rectal contraction type defecation $\left(\mathrm{P}^{*}\right.$ rect) $(\mathrm{A})$, and the second as strain type defecation (Pabd) (B). In both subjects, anal pressure increased mild to moderately. However, the Parkinson's disease patient (C) showed paradoxical sphincter contraction on defecation (Pana) with minimum strain (Pabd), and this patient was almost unable to defecate with large post-defecation residuals.

facilitates the lower GI tract. Centrally administered dopamine D1/D2 receptor agonists ${ }^{15}$ or electrical stimulation of the putamen and the globus pallidus evokes colonic contraction, probably via the Barrington's nucleus. ${ }^{16}$ This nucleus, which is known as the pontine micturition and defecation centre, is 
located in or just adjacent to the locus coeruleus. Electrical or chemical stimulation of the Barrington's nucleus also evokes colonic contraction. ${ }^{16}$ As both nigrostriatal dopaminergic pathways and the locus coeruleus are lesioned in $\mathrm{PD}$, these lesions may result in the decreased colonic contraction in the patients. The peripheral abnormality in PD has been recognised as a decrease of dopaminergic neurons with Lewy bodies in the intrinsic myenteric nerve plexus (Auerbach's plexus) of postmortem PD. ${ }^{2}$

At the resting state, previous studies have shown that the anal pressure of PD patients was low $^{8}$ or normal. ${ }^{17}$ The anal pressure of our patients was also slightly lower than that of control subjects. The resting anal pressure may reflect sympathetic innervation in the internal anal sphincter, as lesions or anaesthesic blocks at T12-L2 (where the sympathetic preganglionic neurons are located) substantially lessen the anal pressure. $^{18}$ Similarly, PD patients have low $^{8}$ or normal ${ }^{17}$ anal pressure increase on squeezing. In this study, the anal pressure increase on squeezing of the PD patients was slightly lower than that of control subjects. However, the external sphincter EMG showed neurogenic change in none of our patients, in agreement with previous reports in which such change occurred in only $0 \%-15 \%$ of $\mathrm{PD}$ patients. ${ }^{11}{ }^{13}$ This result indicates that the sacral Onuf's nucleus is spared in the majority of PD patients. Nevertheless, the latent anal sphincter dysfunction may explain the fecal incontinence that occurs in advanced cases.

During filling, our PD patients had a slightly but not significantly larger rectal volume at first sensation and maximum desire to defecate compared with control subjects, which was in agreement with previous reports. ${ }^{17}$ During filling, the PD patients had the same rectal compliance as control subjects, as reported in a study using the balloon inflation method. ${ }^{17}$ However, our study showed the amplitude of the phasic rectal contraction in the PD patients was less than that in control subjects $(p<0.05)$. The decreased phasic contraction may share the same aetiology with the decrease in colonic transit time. In addition, there was a close relation between the anal and rectal contractions in normal subjects-that is, when the rectal pressure increased, the anal pressure tended to decrease. However, in the PD patients, both rectal and anal pressures tended to increase together. This phenomenon during filling resembles the paradoxical sphincter contraction on defecation (PSD) as described below. Although none of our patients had a history of fecal incontinence, two of the PD patients leaked rectal content, which was never seen in control subjects.

During defecation, it remains a subject of controversy whether true rectal contraction occurs, as abdominal strain is large enough to mask the rectal contraction if present. Previous researches have not measured the differential rectal pressure component, with the exception of a study by MacDonald et $a{ }^{19}$ who did not see a rectal contraction on defecation. However, in our study, four of the control subjects had a rectal pressure increase on defecation, and the others defecated only with abdominal strain. Overall, the rectal pressure increased moderately. However, most PD patients could not defecate completely and had post-defecation residuals of $133 \mathrm{ml}$, which was significantly larger than those in the control group $(p<0.01)$. The abdominal strain and rectal contraction on defecation in the PD patients were less than those in control subjects. The abdominal strain plays physiological parts in both coughing and defecation, which is associated with activation in the brain stem nuclei such as Kolliker-Fuse nucleus and medullary respiratory neurons. ${ }^{20}$ In our study, however, the PD patients showed a less pronounced increase in abdominal pressure on coughing or Valsalva manoeuvre than did the control subjects $(p<0.01)$, as described in a previous report. ${ }^{21}$ The mechanism of the impaired strain in PD may include rigidity and reduced contractility of the axial muscles, and a failure of coordinated glottis closure. ${ }^{21}$ During defecation, the anal pressure increase on defecation in the patients was significantly larger than that in control subjects $(p<0.05)$, with an increase in the sphincter EMG activity. This finding in PD has been described PSD, or anismus, in studies using sphincter $E M G{ }^{713}$ radiography, ${ }^{8}$ and anal pressure measurement..$^{48^{17}}$ Therefore, both weak abdominal strain and PSD seem to be the most important causes of difficulty in stool expulsion in PD patients. The underlying pathophysiology of PSD in PD patients is still uncertain. Mathers et $\mathrm{al}^{7}$ considered PSD a focal dystonia. PSD also occurs in spinal cord injured patients, ${ }^{22}$ suggesting dysfunction in the suprasacral descending pathway to the external sphincter is a contributing factor. Previous studies have shown that apomorphine, a D l/2 receptor agonist, lessened PSD. ${ }^{78}$ This effect was not antagonised by domperidone, which did not penetrate the blood-brain barrier, suggesting the central abnormality may produce PSD.

In conclusion, constipation is a prominent lower GI tract dysfunction in patients with PD. This disorder is characterised by slow colonic transit, decreased phasic rectal contraction, and weak abdominal strain and PSD.

\section{Authors' affiliations}

R Sakakibara, T Uchiyama, M Asahina, T Hattori, Department of Neurology, Chiba University, Chiba, Japan

T Odaka, K Yamaguchi, T Yamaguchi, Department of 1 st Internal Medicine, Chiba University

T Yamanishi, Department of Urology, Dokkyo Medical College, Tochigi, Japan

Competing interests: none declared.

Correspondence to: Dr R Sakakibara, Neurology Department Chiba University, 1-8-1 Inohana Chuo-ku, Chiba 260-8670, Japan; sakaki@med.m.chiba-u.ac.jp

Received 12 March 2002

In final revised form 27 September 2002

Accepted 23 October 2002

\section{REFERENCES}

1 Abbott RD, Petrovitch $H$, White LR, et al. Frequency of bowel movements and the future risk of Parkinson's disease. Neurology 2001;57:456-62.

2 Kupsky WJ, Grimes MM, Sweeting J, et al. Parkinson's disease and megacolon; concentric hyaline inclusions (Lewy bodies) in enteric ganglion cells. Neurology 1987;37:1253-5.

3 Edwards LL, Pfeiffer RF, Quigley EMM, et al. Gastrointestinal symptoms in Parkinson's disease. Mov Disord 1991;6:151-6.

4 Bassoti G, Maggio D, Battaglia E, et al. Manometric investigation of anorectal function in early and late stage Parkinson's disease. J Neurol Neurosurg Psychiatry 2000;68:768-70.

5 Sakakibara R, Shinotoh H, Uchiyama T, et al. Questionnaire-based assessment of pelvic organ dysfunction in Parkinson's disease. Autonomic Neuroscience: Basic and Clinical 2001;92:76-85

6 Wenning GK, Ben-Shlomo Y, Hughes A, et al. What clinical features are most useful to distinguish definitive multiple system atrophy from Parkinson's disease? J Neurol Neurosurg Psychiatry 2000;68:434-40.

7 Mathers SE, Kempster PA, Swash M, et al. Constipation and paradoxical puborectalis contraction in anismus and Parkinson's disease a dystonic phenomenon? J Neurol Neurosurg Psychiatry 1988:51:503-7.

8 Edwards LL, Quigley EMM, Harned RK, et al. Defecatory function in Parkinson's disease; response to apomorphine. Ann Neurol 1993;33:490-3.

9 Devroede G. Radiopaque marker measurement of colorectal transit. In: Schuster MM ed. Atlas of gastrointestinal motility in health and disease. Baltimore: Williams and Wilkins, 1993:57-75.

10 Kamm MA. Pelvic floor tests. In: Kamm MA, Lennard-Jones JE eds. Constipation. Petersfield: Wrightson Biomedical Publishing, 1994:145-53.

11 Palace J, Chandiramani VA, Fowler CJ. Value of sphincter electromyography in the diagnosis of multiple system atrophy. Muscle Nerve 1997;20:1396-403.

12 Brillant P, Pemberton JH. Rectal compliance. In: Smith LE, ed. Practical guide to anorectal testing. 2nd edn. New York: Igaku-Shoin, 1995:227-34

13 Jost WH, Schrank B. Defecatory disorders in de novo Parkinsonians; colonic transit and electromyogram of the external anal sphincter. Wien Klin Wochenschr 1998;21:535-7.

14 Ashraf W, Pfeiffer RF, Park F, et al. Constipation in Parkinson's disease objective assessment and response to psyllium. Mov Disord $1997 ; 12: 946-51$ 
15 Bueno L, Gue M, Fabre C, et al. Involvement of central dopamine and D1 receptors in stress-induced colonic motor alterations in rats. Brain Res Bull 1992;29:135-40.

16 Valentino RJ, Miselis RR, Pavcovich LA. Pontine regulation of pelvic viscera; pharmacological target for pelvic visceral dysfunctions. Trends Pharmacol Sci 1999:20:253-60.

17 Stocchi F, Badiali D, Vacca L, Dlba L, et al. Anorectal function in multiple system atrophy and Parkinson's disease. Mov Disord 2000;15:71-6.

18 Frenckner B, Ihre T. Influence of autonomic nerves on the internal anal sphincter in man. Gut 1976;17:306-12.
19 MacDonald A Paterson PJ, Baxter JN, et al. Relationship between intra-abdominal and intrarectal pressure in the proctometrogram. $\mathrm{Br} J$ Surg 1993;80:1070-1.

20 Iscoe S. Control of abdominal muscles. Prog Neurobiol 1998:56:433-506

21 Fontana GA, Pantaleo T, Lavorini F, et al. Defective motor control of coughing in Parkinson's disease. Am J Respir Crit Care Med $1998 ; 158: 458-64$.

22 Lynch AC, Antony A, Dobbs BR, et al. Bowel dysfunction following spinal cord injury. Spinal Cord 2001;39:193-203.

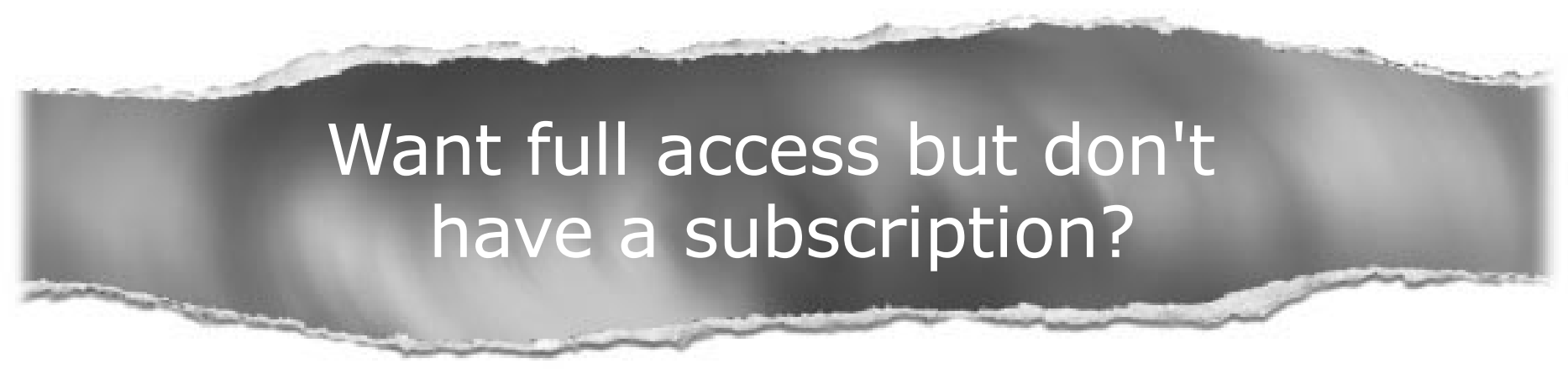

\section{Pay per access}

For just US $\$ 25$ you can have instant access to the whole website for 30 days. During this time you will be able to access the full text for all issues (including supplements) available. You will also be able to download and print any relevant pdf files for personal use, and take advantage of all the special features Journal of Neurology, Neurosurgery, and Psychiatry online has to offer.

\section{www.jnnp.com}

